\title{
Auguste Comte y John Stuart Mill: distancias epistemológicas
}

\section{Lucas Kruzolek \\ Universidad Nacional del Nordeste}

\section{Resumen}

En el utilitarismo inglés de John Stuart Mill y en el positivismo de Auguste Comte pueden encontrarse, en líneas generales, dos de las posiciones filosóficas más cercanas del siglo XIX. El ánimo científico y la ilusión de un progreso social de la mano del desarrollo de las ciencias atraviesan y animan desde el interior la totalidad de sus proyectos. Sin embargo, al interior de sus posiciones epistemológicas persisten distancias insalvables. Lógico uno y matemático el otro, el eje medular de sus diferencias será la determinación del lugar de sus disciplinas nativas en relación al sistema jerárquico de las ciencias. El solapamiento conflictivo entre instrumentos lógicos y matemáticos a la hora de configurar un método científico hace eco, a su vez, de desencuentros más profundos que conectan sus posiciones a la historia más general de las discusiones y de los solapamientos conflictivos de tradiciones epistemológicas enteras.

\begin{abstract}
In John Stuart Mill's English utilitarianism and Auguste Comte's positivism can be found, in general terms, two of the closest philosophical positions of the nineteenth century. The scientific spirit and the illusion of a social progress by the hand of the development of the sciences cross and animate from the interior the totality of its projects. However, insurmountable distances persist within their epistemological positions. Logician the one and mathematician the other, the core axis of their differences will be the determination of the place of their native disciplines in relation to the hierarchical system of the sciences. The conflicting overlap between logical and mathematical tools in setting up a scientific method echoes, in turn, of deeper misunderstandings that connects their positions to the more general history of the discussions and conflicting overlaps of entire epistemological traditions.

Palabras clave: Mill, Comte, epistemología, lógica, matemática.
\end{abstract}




\section{§1 Introducción}

La historia de la crítica comparativa entre los sistemas filosóficos de Auguste Comte y de John Stuart Mill ha enfatizado siempre como principales líneas de rupturas teóricas aquellas discusiones que mantuvieron de manera explícita en sus intercambios epistolares. En ellas se tematizaban cuestiones tales como el problemático lugar de la libertad individual y de los derechos civiles en la sociocracia positivista, en relación con los fuertes tintes despóticos que la caracterizaban; la cientifización de la esfera religiosa y espiritual; o, incluso, la consideración de la naturaleza femenina y de sus respectivos derechos.

El objetivo de mi trabajo será, por el contrario, encauzar la confrontación hacia un área que considero que ha permanecido relativamente soslayada, y donde, sin embargo, existen diferencias más profundas y con mayor fuerza significativa: las distancias presentes entre sus posturas epistemológicas. La motivación y la dirección de la pregunta me fueron señaladas a partir del lugar y la importancia que cada uno otorgaba a los instrumentos lógicos y matemáticos en relación al estudio científico de la naturaleza. Es muy curioso que entre dos de los enfoques epistemológicos relativamente más cercanos del siglo XIX se encuentren, en cambio, en este punto diferencias tan pronunciadas y sugestivas. Además, ciertas lecturas que realicé paralelamente en el cursado de la cátedra de Epistemología y metodología de las ciencias me señalaron una sospecha inicial: que tales diferencias podían significar meras incongruencias superficiales, sino que en cambio podían llegar a explicarse en función de la estructuración histórica del campo y de las tradiciones epistemológicas. En este sentido, a partir del desarrollo del trabajo se irá revelando cómo tales discrepancias, en realidad, hunden sus raíces en profundas distancias epistemológicas.

El enfoque lógico de Mill viene acompañado con un conjunto de fuertes acusaciones a la doctrina de Comte que hacen centro en supuestas inconsistencias e imprecisiones a la hora de brindar criterios rigurosos para la validación de hipótesis científicas. Pero tales irregularidades denunciadas por Mill, como se verá, se encuadran en una ambigüedad mucho más general y que conmociona de manera más 
intensa la continuidad del pensamiento de Comte. Lo que al fin de cuentas sale a la luz es la conflictiva convivencia, al interior de la doctrina de Comte, de dos grandes enfoques metódicos, uno subjetivo y otro objetivo, aparentemente irreconciliables y cuyo antagonismo fue objeto de discusiones entre la mayoría de los positivistas. Una propuesta de reconciliación entre ambos fue planificada por Levy-Bruhl en la obra que aquí se cita.

El trabajo, en síntesis, intentará dar cuentas del siguiente trayecto: voy a tratar de mostrar, en primer lugar, de qué modo las acusaciones epistemológicas de Mill devienen en el planteamiento lógico de sus célebres cánones de inducción; en segundo lugar, poner a la vista la manera en que se incardinan estas antinomias reprochadas por Mill con una contradicción teórica mayor; y por último, a partir de las distancias que originariamente despertaron mi interés -la primacía de la lógica o de la matemática en el sistema de las ciencias- busco esbozar una tentativa de reconciliación, en cierto sentido original, pero aun así deudora en gran medida de los esfuerzos de Levy-Bruhl, para la problemática coexistencia de los métodos objetivo y subjetivo, que me permitan, al mismo tiempo, releer el conflicto en clave histórica, reincorporándolos al interior de la trama más general de las tradiciones epistemológicas.

\section{§2.1 El problema de los criterios}

En su ensayo Auguste Comte and positivism, John Stuart Mill dedica un par de párrafos a tratar ciertas oposiciones existentes entre sus propias consideraciones epistemológicas y ciertas ideas expuestas por Comte en el Curso de filosofía positiva y en obras posteriores. La economía de palabras -si se la compara con la extensión del resto del ensayo- se muestra a tono con la consideración que Mill le otorga a dicha cuestión: las distancias entre sus proyectos no pasan de ser productos de un infeliz pero nada alarmante descuido. No vendría a ser otra cosa que un desarreglo de pequeñas sombras en ese inmaculado panteón de la filosofía que es, a sus ojos, la doctrina positiva del Comte maduro. Esas pequeñas desavenencias no quitan para 
nada que en ese lugar se haya fraguado, fruto de una pericia inédita, un tratado del método y del sistema de las ciencias con grado de refinamiento y precisión sin rival hasta su momento. Aun así, al margen de lo elogios y aunque pueda parecer contradictorio, lo cierto es que un aspecto esencial fue descuidado: el cuerpo de las especulaciones del sistema positivo comteano, si bien legítimo, permanece confinado al tratamiento de los progresos objetivos de las ciencias y a la tematización de la metodología idónea a utilizar para el descubrimiento de nuevos conocimientos, cuando una verdadera filosofía de la ciencia debería dar cuenta con igual rigurosidad también de los criterios lícitos de validación de tales conocimientos.

Como adelantándose a la distinción formulada años más tarde por Reichenbach aquella que distingue entre el contexto de descubrimiento $y$ el contexto de justificación-, las críticas de Mill hacen notar la pobreza de la doctrina comteana a la hora de dar cuanta de las instancias de validación de los conocimientos alcanzados. La delimitación de un órganon de principios formales a través de los cuales se pueda decretar la validez de cualquier inferencia científica, es decir, el perfeccionamiento del sistema de cánones lógicos, no forma parte, como pudiera pensarse, de un mero pasatiempo accesorio a la tarea de las ciencias; todo lo contrario, para Mill, en fiel consonancia con el espíritu anglosajón que lo remonta hasta Bacon, dar cuenta de tales criterios es dar cuenta de un ars artium, es perfeccionar "the science of sciencie itself" ${ }^{\prime 95}$. La razón de ser de la reflexión filosófica sobre las ciencias ha de buscarse, entonces, en la reconstrucción de los criterios formales que fundamenten el ejercicio científico. Pero para que la filosofía pueda revestirse, de una vez por todas, de la seriedad y de las exigencias positivas, no basta con que su campo de acción se circunscriba al interior del despliegue positivo de las ciencias, sino que además ha de entenderse como continuidad de un mismo impulso histórico. Una vez alcanzados los descubrimientos, la pregunta por su validez ha de ser motivada por la fuerza intrínseca del mismo ánimo científico: la necesidad de testear la validez no solo de las conclusiones alcanzadas, sino de todo el proceso inferencial, debe espolear la

\footnotetext{
${ }^{95}$ MILL, John Stuart. A sistema of Logic, rationative and inductive. Nueva York, Harper \& Brothers, 1882, p. 16.
} 
conciencia del científico en virtud de su predisposición natural a la precisión y veracidad.

Por estos motivos, resulta completamente inconcebible para Mill el silencio con el que se oscurecen los criterios de validación en la obra de Comte. Mucho más que una simple incoherencia, lo que seguramente aterró a Mill a la hora de leer los últimos capítulos del Curso fue encontrar lo que para él habrá sido una suerte de atentado o boicot interno a la propia actividad científica y a la nueva filosofía positiva. En esas últimas páginas puede leerse, efectivamente, una suerte de indolencia por parte de Comte con respecto a la importancia de los estudios lógicos. Si son emprendidos en un distanciamiento formal a la propia actividad científica, las discusiones lógicas son, a su juicio, completamente estériles; e incluso mucho más perjudiciales que útiles, en la medida en que avivan el fuero dogmático de la razón, empeñada más en defenestrar los resultados ajenos que en promover al acrecentamiento del patrimonio común de los conocimientos. Toda tentativa de elaboración de una doctrina formal del método, tanto en vistas de sus aplicaciones heurísticas o evaluativas, debe acallarse y disolverse en la marcha positiva de las investigaciones científicas. No hay lugar en el sistema comteano para la figura del filósofo adocenado, ajeno a la actividad científica, que flotando en la trascendencia de sus instrumentos lógicos no se cansa de alimentar su orgullo a costa de despreciar los hallazgos científicos ajenos. La lógica de la ciencia conforma los primeros principios de su actividad efectiva, no los primeros principios de un intelecto virtual, y por lo tanto tiene que ser articulada en el calor de su marcha, en la inmanencia del ejercicio investigativo.

\section{§2.2 La causa del error}

Disolver de esta manera la empresa de la fundación de los preceptos lógicos en la espontaneidad de la actividad científica, únicamente para resguardarse de cierta voluntad normativa malintencionada, podría leerse para Mill como consecuencia de una actitud bastante inocente y temeraria que sobreestima la capacidad autorregulativa y la eficacia de las ciencias. Aun así, en cambio, el principal centro de 
gravedad sobre el cual se entronca y se justifica al mismo tiempo la desidia de Comte tiene que ver más bien con un desacierto en la concepción del objeto de estudio de las ciencias. En lo que Mill está pensando puntualmente es en el giro comteano básico que posiciona como único propósito científico el estudio y descubrimiento de leyes invariables en la naturaleza, y que arroja las preocupaciones por las causas, cargadas de vicios escolásticos, a la esfera de los estadios teológico y metafísico ya superados.

El gesto esencial no es puesto en cuestión: recogiendo la fuente común de ambos en el pensamiento de Hume, no hay nada que objetar en el rechazo a la consideración de causas que, presentándose como realidades subsistentes y meta-fenoménicas, pretenden ser indagadas por el poder limitado de nuestra razón y asentadas como principios explicativos de los fenómenos naturales. El problema no es la impugnación de realidades últimas, que difiriendo cualitativamente de los fenómenos naturales se manifiestan a la inmediatez intuitiva del entendimiento al margen de toda posible instancia de observación fáctica; el problema recae en el abandono apresurado de la palabra causa. Que se eche de menos la rúbrica ontológica, es decir, la consideración de las causas como causas eficientes o causas finales, no implica que debamos renegar del vocablo mismo de causalidad y de su rico importe semántico y reducir todo a cuestiones de sucesiones fenoménicas.

Si nos consagramos a un estudio diligente de las modalidades de sucesión, tal como lo emprendió Mill en su Sistema de Lógica, deberíamos llegar a entrever como él la diferencia medular que en el seno de las sucesiones fenoménicas distingue aquellas invariables de aquellas incondicionales. Por un lado, podemos hablar de un determinado tipo de sucesiones que se presentan a nuestra experiencia bajo las luces de una ley invariable, tal como ocurre si decimos, por ejemplo, "el día sucede a la noche". Pero en estos casos hallamos, además, que el consecuente no se deja percibir libre de ciertas condiciones que recaen más allá de las denotadas por el antecedente original; condiciones que, en un nivel distinto, conciertan incluso la posibilidad de la sucesión original: el acontecer tanto del día como de la noche, tomados por separado y al mismo tiempo en su entramado secuencial -es decir, el acontecer del día y de la 
noche, pero también el acontecer de la sucesión misma-, está sujeto a su vez a la concurrencia de otros fenómenos, como por ejemplo, en este caso, la rotación terrestre. En este sentido puede decirse de la imbricación fenoménica rotación terrestre-acontecer del día y de la noche que, a diferencia de la sucesión original díanoche, el carácter de la uniformidad de su sucesión no es únicamente invariable sino también incondicional.

¿Por qué es importante para Mill esta precisión? Por el hecho de que a través de ella se vuelve transparente un desborde del concepto de ley de sucesión: se comprueba que las conexiones que ligan ambas series fenoménicas en sí no poseen la misma intensidad veritativa. La ley de sucesión de la rotación terrestre mantiene una correspondencia subsidiaria con la sucesión del día y la noche. Y sucede así porque ella no expresa una mera coincidencia fáctica cuya invariabilidad se sostiene, por el momento, frente a las contrastaciones; en su formulación se enlaza, en cambio, una relación inmediata, y por lo tanto aséptica de condiciones: se enuncia una ley de causación. De esta forma, con la rehabilitación del concepto de causa, se está jugando la clave de acceso a una crítica profunda a las concepciones epistemológicas de Comte.

\section{§2.3 De la ley de causación a los cánones de inducción}

En la fractura anterior no solamente se dibuja una disociación y un apartamiento; más importante aún es el desplazamiento de fundamentación que pone a la base de toda ley fenoménica, sea de sucesión o de coexistencia, la universalidad de la ley de causación, según la cual dado un fenómeno $x$ existe siempre otro fenómeno 0 conjunción de fenómenos de los cuales $x$ es consiguiente de manera invariable e incondicional. Dicha ley, a su vez, apunta hacia un fundamento más profundo, sedimentado como condición de posibilidad de toda referencia científica a la experiencia, y que Mill formula con el nombre de principio de la uniformidad de la naturaleza. El principio reza: lo ocurrido una vez volverá a ocurrir siempre que exista un grado de similitud suficiente en las circunstancias. El asentimiento al principio conforma el fundamento último de toda conclusión obtenida por inducción. 
De igual modo, es crucial que no pasemos por alto el énfasis de Mill en este principio, ya que en su hipotético rechazo se va de lleno la posibilidad de todo conocimiento positivo. A la par que toda inducción encuentra su suelo en el postulado de la uniformidad, debemos tener en cuenta que para Mill, así como para toda la corriente empirista inglesa en la que se enmarca su pensamiento, todo conocimiento recogido en nuestro trato inmediato con lo empírico es particular, y los grandes postulados generales sobre los cuales puede operarse un silogismo que infiera conclusiones de manera deductiva son consecuencia de una maniobra inductiva previa. Toda ley científica, por lo tanto, incluso aquellas en las que resuenan los clamores de máxima necesidad y universalidad: las que conforman el órganon de proposiciones matemáticas y geométricas -tan caras a Comte-, tienen su raíz en inducciones empíricas, y la evidencia y consistencia de sus verdades no dependen de su naturaleza constitutiva sino del asentimiento al postulado de uniformidad.

De esta forma se justifica el hecho de que la apuesta lógica de Mill por proyectar un sistema de criterios que decida la legitimidad formal de los descubrimientos científicos y que complemente la obra de Comte, termine derivando en la confección de sus célebres cánones de inducción. Con ellos se ve posibilitada la introducción, en el sistema de las ciencias, de los instrumentos necesarios para emprender la evaluación de sus conclusiones, y para disipar, en consecuencia, las vagas sombras que acechaban en el Curso cuando se trataban de tales cuestiones.

En efecto, Mill no se guarda de constatar las imprecisiones y ambigüedades de Comte a la hora de definir un criterio específico para la validación de hipótesis. En un primer momento el nuevo espíritu científico parecería estar caracterizado por cierta aspiración vehemente de rigurosidad, que mira con malos ojos toda tesis que no se haya mostrado acorde con los hechos, e incluso que no haya probado que su falsedad misma es inconsistente con la experiencia. Pero avanzando en el Curso uno se encuentra con apreciaciones a primera vista bastante incompatibles, según las cuales una impecable objetividad no es condición indispensable para la aceptación de una hipótesis; basta que ella satisfaga cierto anhelo "natural" del intelecto hacia la síntesis 
y la simplicidad, o que, aun pudiendo ser falsas, no violenten en tan alto grado la realidad exterior, sino que hasta contribuyan a la elaboración de teorías más acertadas. Pero aun así, más allá de estos puntos, sin duda la expresión más clara de inconsistencia, y donde Mill advierte una deflación extrema de la rigurosidad científica, se percibe en aquellos momentos en los que, enfatizando la naturaleza relativa del espíritu positivo, Comte subordina la validez de los descubrimientos "al conjunto del progreso social" ${ }^{\prime 96}$ y a su capacidad de satisfacer las exigencias de previsión racional, levantando como cima de todos los criterios epistemológicos un destino puramente subjetivo: "This notion of the "destination" of the study of natural laws is to our minds a complete dereliction of the essential principles which form the Positive conception of science; and contained the germ of the perversion of his own philosophy which marked his later years" ${ }^{\prime 97}$.

\section{§2.4 Mucho más que un problema de criterios}

Este tipo de incongruencias, que tocan a los criterios de validación de las hipótesis científicas y, con ello, a la consideración del valor de objetividad de las leyes naturales, despertaron en su tiempo el escrúpulo de fieles seguidores de la doctrina positivista como Mill-que, lejos de encontrar una resolución conveniente, prolongaron el debate durante los años siguientes. En adelante, estas discusiones epistemológicas se enmarcaron en el problema, más amplio, de la delimitación de la naturaleza del método positivo en general, hecho que llegó a conmocionar la entera uniformidad de la obra filosófica de Comte. Para Levy-Bruhl ${ }^{98}$, el problema del primado de instancias de validación objetivas o subjetivas en el método positivo despertó acaloradas discusiones entre los comentadores que, en todos los casos, no habiendo podido perfilar una conciliación adecuada, no vieron otra salida que la de remitirse a la existencia de dos métodos antagónicos, cuyo solapamiento encontraría su razón de ser en un progresivo declive intelectual del maestro. Si bien es cierto que los años despertaron en Comte cierta afección hacia temas cada vez más ajenos a sus

\footnotetext{
${ }^{96}$ COMTE, Auguste. Discurso sobre el espíritu. Madrid, Biblioteca Nueva, 1999, p. 79.

${ }^{97}$ MILL, John Stuart. Auguste Comte and positivism. Londres, N. Trabürner \& Co., 1865, p. 62.

${ }^{98}$ Cfr. LEVY-BRUHL, Lucien. The philosophy of Auguste Comte. Londres, Swan Sonnenschein \& Co, 1900.
} 
teorizaciones científicas iniciales, las indecisiones y ambigüedades denunciadas por Mill ya se estarían presintiendo "en germen" en los tratados del Curso.

Desarrollando una serie de argumentos intrínsecos o de orden lógicoepistemológico, por un lado, y de carácter moral o religioso, por otro, Levy-Bruhl propone en un capítulo de su obra The philosophy of Auguste Comte una tentativa de reconciliación entre ambos métodos antagónicos. A su entender, para Comte la unidad sistemática de la filosofía positiva no puede aspirar a ser concretada desde un punto de vista objetivo, ya que esto significaría sentar como propósito una quimera: lograr reducir la multiplicidad de los fenómenos naturales a una única ley. Lo que implicaría no solo una sobrevaloración ingenua de nuestras capacidades cognitivas, sino también la muerte de toda investigación futura. La mente humana, una vez arraigada en el estadio positivo, tiene plena consciencia de sus límites, y reconoce que la síntesis metódica no puede jamás componerse a partir de las observaciones empíricas; es decir, desde una consideración en la que el primado de la observación enlace el itinerario de los conocimientos desde el Mundo hacia el hombre. Por el contrario, una vez fundada la sociología, la plena concreción del sistema positivo deja en claro que la síntesis anhelada ha de ser buscada en la opuesta visión de un método subjetivo, cuya primacía nace ahora en el hombre y se proyecta hacia el Mundo, pero no de la manera en la que podría proyectarse un teólogo o un metafísico, sino cargando en sus manos los principios científicos conquistados en el estadio positivo.

A pesar de su integridad general, la conciliación ensayada por Levy-Bruhl no parece echar luces claras en la materia específica de los criterios de verificación y sus consecuencias epistemológicas que estamos discutiendo. La suerte de vuelta objetivada a un método subjetivo ¿quiere decir que de las inconsistencias presentadas en el Curso se deberá optar por aquellas que juzgan el valor de las hipótesis científicas en virtud de su capacidad predictiva, sintética y estética? Y si esto es así ¿implica con ello que en este tribunal de corte subjetivo, o intersubjetivo en el mejor de los casos, se desoiga cualquier precepto lógico o fáctico con aspiraciones de objetividad? O si, como parece ser la consecuencia más aceptable, la reconciliación implica una 
convivencia pacífica entre sendos grupos de criterios, ¿cuál sería el carácter de esta asociación? ¿existen condiciones suficientes, o más bien en la validación de las leyes la satisfacción de cada uno de ellos es necesaria? Y por último ¿cómo conciliar la mentada invariabilidad de las leyes científicas, sobre la cual descansa la medida de su previsión racional, con la conciencia de la relatividad del conocimiento, de la finitud de nuestro entendimiento y de la complejidad irreductible de los fenómenos? La ausencia de respuestas no se despierta en la postura de Levy-Bruhl como consecuencia de cierto extravío teórico, sino que sus reservas no son más que un mero espejismo motivado por la generalidad de su mirada.

\section{$\S 2.5$ Elementos para una reconciliación}

Para asimilar mejor la continuidad que enlaza simétricamente y en una unidad no antagónica ambos principios metódicos, es necesario que posicionemos como eje de análisis las observaciones que realiza Comte sobre del carácter de las matemáticas y su lugar en el sistema de las ciencias y en el entramado conceptual de la filosofía positiva. El movimiento de disolución de un método objetivo inicial en los controvertidos cánones intersubjetivos que tanto desazonan a Mill y al resto de los positivistas, puede leerse en clave de otro movimiento: aquel que disuelve, del mismo modo, una supremacía originaria de las matemáticas en el método y el sistema de las ciencias, en una ulterior preeminencia de la física social. Pero si en nuestras intenciones está salvar la homogeneidad de la doctrina positiva, creo que hay que dejar de ver en este conflicto la historia de la fricción agónica de un binomio irreconciliable, que se resolvería finalmente en un trasvase valorativo de un polo a otro. Una lectura más acorde debería, por el contrario, desglosar, en el estudio de las matemáticas, la ciencia de lo que en algunos lugares es presentando como espíritu, para encontrar en este último el vehículo del acuerdo y la síntesis entre todas las partes del sistema de las ciencias. Únicamente la confusión entre la ciencia de la matemática, que como tal ocupa el primer escalón del edificio científico, y el espíritu matemático, que se posiciona, en cambio, como fundamento de todo el sistema, recorriéndolo en su 
integridad a la manera de un pneuma vital; únicamente esta confusión, digo, puede fundar la posibilidad de una interpretación disruptiva y heterogénea del pensamiento de Comte.

Una vuelta a las polémicas entabladas con Mill nos permitirá articular con exactitud esta distinción. En ciertos capítulos del Curso, y posteriormente de manera explícita en el Discurso sobre el espíritu positivo, parece que existen, sino cercanías, al menos intentos de aproximaciones teóricas por parte de Comte. Precisamente, el capítulo V del Curso, dedicado a la exposición de las ciencias biológicas, es uno de los lugares donde Comte enuncia con mayor claridad lo que él considera el "dogma fundamental" de la ciencia y de la filosofía positiva; dogma según el cual "the rational study of nature procedes on the ground that all phenomena are subject to invariable laws, which it is the business of philosophical speculation to discover" ${ }^{\prime 99}$. Si bien el estado actual de los desarrollos científicos descubren a la mayoría de las ciencias en una situación de relativa juventud, es una condición necesaria para la posibilidad del despliegue de su investigación mantener la convicción de que la totalidad de los fenómenos naturales se hallan entrelazados por leyes susceptibles de ser algún día reveladas ¿Cómo hacemos para no encontrar aquí una contradicción a lo que antes se había dicho? La finitud del entendimiento humano y la irreductible complejidad de los fenómenos eran contraargumentos que Comte presentaba frente a los proyectos que buscaban fundamentar la validez de las leyes científicas. De lo que se trata ahora, en cambio, no es de imaginar un sedimento ontológico que resuelva, a partir de una correspondencia recíproca, la posibilidad y la validez del conocimiento científico; sino, en cambio, de proyectar algo así como un horizonte normativo que inaugure y alimente el despliegue científico.

La confianza que anuncia la presencia virtual de una explicación omnicomprensiva es fuertemente compatible con aquel principio de uniformidad sobre el cual Mill hace descansar toda inferencia inductiva. En efecto, especular con una naturaleza enteramente legaliforme significa tramitarla en términos de uniformidad. Y, más aún,

\footnotetext{
${ }^{99}$ COMTE, Auguste. The positive philosophy. Tomo II. Londres, George Bell \& Sons, 1896, p. 32.
} 
la cercanía se intensifica en el momento en que Comte postula como fuente de su dogma nada menos que una inducción gradual, al mismo tiempo individual y colectiva.

La visión positiva dista mucho de ofrecerse al espíritu de manera innata; antes bien, la humanidad ha debido sortear las inclemencias de los estadios teológico y metafísico previos para alcanzar la creencia firme en el orden invariable de la naturaleza y en su expresión genuina en leyes de sucesión y coexistencia. Comte va incluso un poco más lejos que Mill, y no solo hace reposar la esencia del ejercicio científico en un dogma inductivo; llega a considerar que la solidez de esta inducción goza de un importante apoyo práctico: el espíritu positivo conquistó su fortaleza gracias al vigoroso impulso que supuso la institución de la astronomía matemática, es decir, con el hecho de que por primera vez un orden importante de fenómenos fue reducido a sus correspondientes leyes científicas, pudiendo establecerse con seguridad la predicción de sus comportamientos.

Pero si en el sistema positivo de Comte, al igual que en los escritos lógicos de Mill, la rigurosidad científica señala como su fundamento la estela de una inducción ¿por qué, en cambio, la armazón de los criterios epistemológicos, aquellos que sentencian o no la cualidad de ley de una hipótesis científica, no coincide en la teoría de Comte con un catálogo de cánones de inducción? ¿Cuál es la razón de que las determinaciones epistemológicas de las leyes científicas permanezcan en tan alto grado de imprecisión, pendulando entre tribunales objetivos e intersubjetivos?

La clave para la resolución de estas aparentes aporías depende de que se pueda distinguir una oposición esencial. La diferencia crucial en este momento es aquella entre, por un lado, el dogma inductivo, que proyectando la uniformidad a la totalidad de la naturaleza alimenta el sueño científico; y por otro un principio inédito, exclusivo de la teoría de Comte, que de alguna manera, desplazando al dogma inductivo, se posiciona como verdadero suelo epistemológico de la actividad científica y que atañe directamente al carácter de las leyes. La distinción que se alcanza es entre, por un lado, un movimiento de fundamentación de la posibilidad lógica de la ciencia, que implica como necesidad la convicción de uniformidad natural, y por otro, un 
movimiento de fundamentación de la rigurosidad y la invariabilidad de las leyes científicas, que señala, esta vez, hacia su carácter matemático. Una ley científica encontrará asegurada tanto su necesidad como su universalidad en la medida en que se encuentre formulada bajo las luces de la abstracción y la simplicidad propias de toda proposición matemática. Por tal razón que "it is only through Mathematics that we can thoroughly understand what true science is" ${ }^{\prime 100}$.

\section{§2.6 La abstracción matemática como esencia del espíritu positivo}

Que la absoluta determinación científica se fundamente en el carácter matemático de sus leyes no significa que cada una de las ciencias deba enarbolar como máxima aspiración la traducción de sus leyes en funciones algebraicas o relaciones geométricas. Es necesario exorcizar cualquier matematismo ingenuo, puesto que la preeminencia aquí no es de la ciencia matemática sino del espíritu matemático. Volcarnos a una interpretación en estos términos sería desoír el meticuloso tratamiento que realiza Comte de los métodos propios de cada ciencia. En tales puntos se deja bien en claro de qué manera la complejidad creciente de los fenómenos naturales exige una metamorfosis metódica en cada área científica. Así, si bien el aparato conceptual aritmético-algebraico traspasa el continuo científico, en cada nivel fenoménico ceden parte de su influencia en manos de instrumentos metódicos propios de cada ciencia, motivados por irrupciones cualitativas que desbordan la finitud de nuestro entendimiento.

La determinación matemática de las leyes no se juega en afinidad con la prepotencia de un dominio cuantitativo por sobre uno cualitativo. La pretensión de esta reducción, es decir, de la posibilidad de una traducción categórica de la totalidad fenoménica en relaciones cuantitativas, coincide, o mejor se enmarca, en los alcances del principio de uniformidad. Para el principio que nos interesa, en cambio, lo que se pone en ejercicio es la coordinación de las leyes científicas con una esfera de abstracción. Es en términos de un nivel de máxima abstracción posible donde debe

${ }^{100}$ COMTE, Auguste. The positive philosophy. Tomo I. Londres, George Bell \& Sons, 1896, p. 60. 
rastrearse el espíritu matemático, y por lo tanto es en virtud de la abstracción el hecho por el que la operación de una ley científica puede revestirse con atributos universales y necesarios: "Here alone [en la Matemática] we find in the highest degree simplicity and severity of scientific law, and such abstraction as the human mind can attain. Any scientific education setting forth from any other point, is faulty in its basis" ${ }^{101}$.

Cuando Comte nos dice que la trascendencia de la astronomía recae en su incorporación al método positivo de la instancia de observación, a lo que se refiere es a la práctica del contraste fáctico. No quiere para nada significar que la ciencia que le antecede, la matemática, no gozase de confrontaciones objetivas. Como ciencia nodriza, ella no puede reducirse a ser un mero juego de la imaginación; la diferencia es que en ella la instancia objetiva se corresponde con una experiencia abstracta. Puede que sus presupuestos estén incardinados en una inferencia inductiva previa, pero lo que no podemos aceptar en la visión de Comte es que la universalidad y la necesidad de sus proposiciones pendan como de un delgado hilo de la aceptación universal y necesaria del principio de uniformidad. La experiencia abstracta es la experiencia en abstracto de la invariabilidad de nuestros principios intelectivos; como experiencia transparente, en bruto, del mecanismo deductivo, constituye la máxima experiencia del poder de nuestra razón, que nos devuelve, en la más profunda inmediatez, una confianza plena de nuestro potencial analítico. El deslumbramiento frente a la experiencia abstracta inaugura una mediación en la empresa de fundamentaciones epistemológicas ausente en Mill. Al no haber palpado la intrínseca precisión y entereza del despliegue deductivo que precede, en todo silogismo científico, a una intuición empírica, Mill retornó la impavidez de su mirada inglesa a la inferencia originaria, y trató de encontrar en la relación con la facticidad el suelo firme para toda empresa científica.

Habiendo alcanzado la certeza de la invariabilidad de las leyes científicas en la universalidad y necesidad de nuestras inferencias abstractas, queda en claro la negativa expresa de Comte hacia la formulación de criterios formales, con los cuales se

${ }^{101}$ COMTE, Auguste. The positive philosophy. Tomo II. Londres, George Bell \& Sons, 1896, p. 60. 
pretenda, una vez determinados los principios supremos de nuestro entendimiento, configurar una inquisición lógica para la validación de hipótesis científicas. Un estudio fructífero de los principios de la actividad científica que delinee la comunión sintética de su método y de su espíritu no debe entablarse en la lejanía lógica de Mill, que intentando hablar por la ciencia solo consigue evocar fantasmas y sucedáneos, sino que debe emplazarse en el vértigo de su marcha misma. Antes que en una inmovilidad abstracta, la lógica positiva se gesta en la actualización de la abstracción.

En el develamiento de su esencia abstracta, el espíritu matemático, como verdadero espíritu positivo, se eleva por sobre cualquier inconsistencia y por sobre cualquier fricción dicotómica. Método objetivo y método subjetivo no son ya dos caras irreconciliables, puesto que tienen asegurada su conciliación en el espíritu matemático. La rigurosa necesidad y universalidad reclamadas desde un enfoque objetivo por positivistas como Mill se hallan garantizadas por la infalibilidad de nuestro potencial analítico. Al mismo tiempo permanece intacta la relatividad social de nuestros conocimientos, no dejándose cegar el espíritu por quimeras que ambicionan concretar la síntesis en la esfera objetiva (una única ley para todos los fenómenos), y en su lugar poniendo las intenciones de su esfuerzo en alcanzar los beneficios predictivos de la ciencia. Ni el estado y ni el orden actual de los conocimientos gozan de una inmediata evidencia, así como tampoco la posibilidad a futuro de develar el plexo de leyes que rigen la totalidad de los fenómenos. Incluso, ni siquiera el hecho mismo de que la suma de los hechos naturales acaece según leyes invariables se presenta a nosotros como una verdad autoevidente. El total de los conocimientos alcanzados, junto con el dogma de la uniformidad que reposa a su base, si no mantienen arraigada su necesidad en la necesidad del espíritu matemático, es decir, si en su carrera por la universalidad no guardan de remitirse a la objetividad de la experiencia abstracta, no cabe otra cosa que esperar que se esfumen en un conjunto de meras expectativas.

\section{§3 Conclusión}


Bastante popular se ha vuelto el carácter abiertamente reaccionario en todos los sentidos de Comte, a punto que en cualquier exposición sistemática de su pensamiento nunca faltan reseñas, por ejemplo, a su oposición a los movimientos revolucionarios. En este sentido, hubiera sido realmente incomprensible que un espíritu como el suyo, que aboga por la seguridad de un progreso gradual y templado con firmeza, se aventurase a sentar como principio de toda la empresa científica una rápida y temeraria inducción como la de Mill. Más acorde a su personalidad se presenta la conclusión a la cual arribamos en este escrito: a saber, que la base de todo ejercicio científico, así como la unidad sintética de su método y de su espíritu, se construyen en torno a las certezas brindadas por las abstracciones matemáticas.

Lejos de formular grandes principios dogmáticos o de describir arriesgados saltos inductivos, la actividad científica se consagra con serenidad a los pequeños hallazgos, apropiándose poco a poco de las verdades naturales. Sus pasos beben la confianza directamente de la gimnasia abstracta del intelecto, que deja traslucir su máxima potencia lógica en la necesidad y universalidad de las operaciones matemáticas. La consideración de la naturaleza de las matemáticas -siempre y cuando se acierte a distinguir sus características como ciencia de su espíritu intrínseco- y su contraposición a arquitecturas lógicas formales suponen una maniobra clave en una reconstrucción de la propuesta epistemológica de Comte que anhele componer y armonizar los aparentes antagonismos.

Para que los resultados del trabajo sean más fructíferos, y para que no se reduzca todo a una discusión antediluviana y casi fosilizada, en mi opinión, se deberían intentar encuadrar las conclusiones en el marco de los estudios, mucho más contemporáneos, de epistemología comparada. De este modo, las distancias epistemológicas entre Comte y Mill podrían leerse a la luz de las distancias históricas y mucho más generales entre los distintos enfoques que orientan y estructuran el campo epistemológico. Estoy pensando particularmente en distinciones entre lineamientos epistemológicos analíticos y continentales (Gutting), o entre tradiciones anglosajonas y francesas 
(Marí). Si bien para Gutting ${ }^{102}$ la separación estricta se empieza a gestar con posterioridad a la Primera Guerra y de la mano del giro lingüístico; y aunque, además, para Marí ${ }^{103}$ la estructuración positiva de las ciencias en la doctrina comteana consuma un proyecto cientificista, inaugurado ya por Bacon y Sant-Simon, que nutrirá con posterioridad y principalmente a los enfoques triunfalistas anglosajones; aun así, creo, las conclusiones de este trabajo deben mostrar la presencia ya rudimentaria de la escisión posterior del campo epistemológico.

Y esto sobre todo y más que nada a partir de la composición plástica de la obra de Comte, a caballo realmente entre ambas tradiciones. Sus reproches anti-metafísicos generales cruzaron el canal y fueron retomados, a grandes luces, por representantes del inductivismo británico, como se vio en el caso de Mill, o posteriormente por el pragmatismo norteamericano y, sobre todo, por el empirismo lógico del Círculo de Viena. Sin embargo, la demarcación del dogma inductivo como principio rector normativo y fundante, y no como proposición ontológica, así como también el posterior énfasis en las experiencias analíticas del espíritu matemático, parecerían ser resonancias de la posterior deflación intuicionista del falsacionismo popperiano.

Para el Círculo de Viena, la objetividad de los enunciados científicos descansa en la condición de verificabilidad empírica propia del uso eminentemente enunciativo del lenguaje; por lo tanto, "algo es "real" en la medida en que se incorpora a la estructura total de la experiencia” ${ }^{104}$. Para el pragmatismo norteamericano de Peirce, la objetividad del método científico se funda, en cambio, en la remisión ontológica a una permanencia externa que se reconstruye, en cambio, intersubjetivamente: "el auténtico origen del concepto de realidad muestra que el mismo implica esencialmente la noción de comunidad"105. Por último, en el racionalismo crítico de Popper, la condición de verificabilidad de los conocimientos científicos también se reconduce desde un presupuesto ontológico último y una correspondiente

\footnotetext{
${ }^{102}$ Cfr. GUTTING, Gary. Continental philosophy of science. Cornwall, Blackwell Publishing Ltd., 2005.

${ }^{103}$ Cfr. MARÍ, Enrique. Elementos de epistemología comparada. Buenos Aires, Puntosur S.R.L., 1990.

${ }^{104}$ Cfr. NEURATH, Otto, CARNAP, Rudolf y HAHN, Hans. La concepción científica del mundo: el Círculo de Viena. En: Redes. Volumen 9, № 18, Junio de 2002, p. 114.

${ }^{105}$ PEIRCE, Charles. Obra filosófica reunida. Tomo I. México, F.C.E., 2017.
} 
enunciación empírica exhaustiva, hacia el mismo carácter intersubjetivo y contrastable del lenguaje y de la experiencia científica: "la objetividad de los enunciados científicos descansa en el hecho de que pueden contrastarse intersubjetivamente" ${ }^{106}$.

Pero la dicotomía de los métodos subjetivo y objetivo en el pensamiento comteano, como vimos, no se resuelve de la misma manera que en los enfoques de la tradición anglosajona o analítica. Se desestima cualquier aspiración exhaustiva y cualquier fondo metafísico, pero el sostén de la objetividad de los enunciados se busca en la contrastabilidad intersubjetiva de la experiencia no de los enunciados, de un particular uso del lenguaje o de la recreación de los efectos empíricos que ellos enuncian, sino de la experiencia abstracta de la matemática. Si bien se vuelve contra las ejecuciones triunfalistas de arriesgados saltos inductivos, no quiere decir tampoco que la seguridad alcanzada en el desarrollo del potencial analítico del entendimiento signifique emular el gesto falsacionista promoviendo, en cambio, operaciones deductivas. Si entroncamos geográfica y teóricamente a Comte con las direcciones generales de la tradición epistemológica francesa, al mismo tiempo tenemos que encarar la disolución de los métodos objetivo y subjetivo al margen de cualquier jerarquización operativa entre inducciones, deducciones o abducciones.

En cambio, en mi opinión, la comprensión íntegra de todo lo que implica al interior del sistema la propuesta del espíritu matemático, se podría alcanzar si se emprende la caracterización del último de los tres estadios comteanos en línea con los rasgos del último de los tres estadios bachelardianos. En este sentido, el ejercicio científico que no persiga su propia muerte persiguiendo un ideal exhaustivo, debe alcanzar la estabilidad y la seguridad de su despliegue no a partir de disquisiciones ontológicas sino a partir de la práctica creativa de una consciencia abstracta. Más allá de la estructuración lógica o formal de las operaciones del método, la fundamentación matemática implica atender, al interior del método, a la operación misma, es decir, a la capacidad de la operatividad como capacidad reactualizante o inventiva. De este modo, nuevamente, la actividad científica no busca reorganizar sus fuerzas a partir de

${ }^{106}$ POPPER, Karl. La lógica de la investigación científica. Madrid, Tecnos, 1980, p. 43. 
reglamentaciones lógicas externas, sino reestructurarse continuamente desde fuerzas inmanentes, en el calor de su marcha, gestionando el metabolismo imposible de un perpetuum mobile. 